\title{
Assessment of Regional Bone Density in Fractured Vertebrae Using Quantitative Computed Tomography
}

\author{
Hany A.G. Soliman ${ }^{1-4}$, Jean-Marc Mac-Thiong ${ }^{1,2,3}$, Annie Levasseur ${ }^{2}$, Stefan Parent ${ }^{1,2,3}$, Yvan Petit ${ }^{2,5}$ \\ ${ }^{1}$ Department of Surgery, University of Montreal, Montreal, QC, Canada \\ ${ }^{2}$ Research Center, Hôpital du Sacré-Coeur de Montréal, Montreal, QC, Canada \\ ${ }^{3}$ Research Center, CHU Sainte-Justine, Montreal, QC, Canada \\ ${ }^{4}$ Al Zahraa Univeristy Hospital, Al Azhar University, Cairo, Egypt \\ ${ }^{5}$ Mechanical Engineering Department, École de Technologie Supérieure, Montreal, QC, Canada
}

\begin{abstract}
Study Design: Cohort study.
Purpose: The aim of this study is to propose and evaluate a new technique to assess bone mineral density of fractured vertebrae using quantitative computed tomography (OCT).

Overview of Literature: There is no available technique to estimate bone mineral density (BMD) at the fractured vertebra because of the alterations in bony structures at the fracture site.

Methods: Forty patients with isolated fracture from T10 to L2 were analyzed from the vertebrae above and below the fracture level. Apparent density (AD) was measured based on the relationship between OCT images attenuation coefficients and the density of calibration objects. $A D$ of 8 independent regions of interest (ROI) within the vertebral body and $2 \mathrm{ROI}$ within the pedicles of vertebrae above and below the fractured vertebra were measured. At the level of the fractured vertebra, AD was measured at the pedicles, which are typically intact. $A D$ of the fractured vertebral body was linearly interpolated, based on the assumption that $A D$ at the fractured vertebra is equivalent to the average $A D$ measured in vertebrae adjacent to the fracture. Estimated and measured $A D$ of the pedicles at the fractured level were compared to verify our assumption of linear interpolation from adjacent vertebrae.

Results: The difference between the measured and the interpolated density of the pedicles at the fractured vertebra was 0.006 and $0.003 \mathrm{~g} / \mathrm{cm}^{3}$ for right and left pedicle respectively. The highest mean AD located th the pedicles and the lowest mean AD was found at the anterior ROI of the vertebral body. Significant negative correlation exist between age and AD of ROI in the vertebral body. Conclusions: This study suggests that the proposed technique is adequate to estimate the $A D$ of a fractured vertebra from the density of adjacent vertebrae.
\end{abstract}

Keywords: Spinal fractures; Bone density; Tomodensitometry

\section{Introduction}

Thoracic and lumbar fractures represent a major health problem all over the world with considerable medical, social and financial implications, because of the abrupt change in the quality of the patient's life, with significant

\footnotetext{
Received May 24, 2016; Revised Jul 8, 2016; Accepted Jul 24, 2016

Corresponding author: Yvan Petit

Mechanical Engineering Department, École de Technologie Supérieure,

1100, Notre-Dame West, Montreal, QC H3C 1K3, Canada

Tel: +1-514-396-8691, Fax: +1-514-396-8530, E-mail: yvan.petit@etsmtl.ca
} 
impact on the ability to work, perform sports, and with the risk of chronic pain [1-6].

It is well known that subjects with poor bone quality are at increased risk of sustaining a vertebral fracture $[7,8]$. Bone strength is determined by a combination of bone size, shape and material properties. In the absence of an accurate single measure of overall bone strength, bone mineral density (BMD) is the most common tool used to assess bone strength indirectly $[9,10]$.

Non-invasive assessment of vertebral BMD can help to choose the best management modality for the patients to reduce bone loss, increase BMD and reduce the risk of fractures. The current clinical standard for BMD assessment is using dual energy X-ray absorptiometry (DXA) $[11,12]$. Unfortunately, DXA does not account for regional variability in vertebral bone density and may include structures that do not add to the mechanical strength of the vertebra, including posterior elements and osteophytes $[13,14]$.

Quantitative computed tomography (QCT) has several advantages over DXA for measuring bone density. QCT allows three dimensional density measurements without superimposition of other tissues with separate analysis of apparent density (AD) of the trabecular and cortical bone. Indeed, previous studies $[13,14]$ showed that spine trabecular bone was more metabolically active than cortical bone and other studies [15] showed that bone strength is significantly more sensitive to a loss of trabecular bone compared with a similar overall reduction in bone mass.

Investigators have previously focused on the measurement of BMD to predict fracture risk and they showed that there is a definite relationship between the change in BMD and fracture risk. Moreover, most of their studies focused only on osteoporotic fractures [7-9,16,17].

There is no available technique to estimate BMD at the fractured vertebra because of the alterations in bony structures at the fracture site.

Knowing the regional variation of density in the fractured vertebra may also help to understand the mechanism of fractures under various loading conditions and the propensity for different fracture patterns. In addition, relating the regional density of the vertebra to the pattern of the fracture could help clinicians to determine the stability of the fracture for each patient and therefore guide the treatment towards non-surgical or surgical management.

The aim of this study is to propose and evaluate a new technique to assess the bone density at the fractured ver- tebra for patients with vertebral fractures. In addition, the regional variation in density of fractured vertebrae will be assessed in an attempt to better understand the characteristic patterns seen in thoracolumbar fractures.

\section{Materials and Methods}

\section{Subjects}

A retrospective cohort of 40 patients (23 male and 17 females) with isolated traumatic thoracolumbar fracture from T10 to L2 aged 20 to 55 years old (36 \pm 7$)$ were included in the study. The study was approved by the Institutional Review Board of the authors' institutions.

\section{Method of measurement}

$\mathrm{AD}$ was measured from QCT at the vertebral body and pedicles of vertebrae above and below the fracture, and at the pedicles of the fractured vertebra which remained intact in all our patients.

For each patient, the fractured vertebra as well as a minimum of two supra- and infra-adjacent vertebrae were imaged upon admission to the hospital using a Lightspeed VCT (GE Medical System, Milwaukee, WI, USA) CT scanner. Four calcium hydroxyapatite calibration standards of known density were also scanned with the same acquisition protocol in order to obtain a correlation between the X-ray attenuation coefficient, expressed in terms of Hounsfield units (HU), and the BMD. The four calcium hydroxyapatite calibration objects had mineral contents of 100, 400, 1,000, and $1,750 \mathrm{mg} / \mathrm{cm}^{3}$ (CIRS Inc., Norfolk, VA, USA) encompassing the common density range of human cancellous and cortical bone.

Measurement of $\mathrm{AD}$ was done using a semi-automatic segmentation software (SliceOmatic, Tomovision, Montreal, QC, Canada). First, the four calibration objects were identified in computed tomography (CT) images and their respective attenuation coefficients were averaged over all the axial CT images. A linear relationship between the calibration objects' average attenuation coefficients and their known mineral density was obtained using linear regression analysis. Matlab software (Matworks, Natick, MA, USA) was used to treat the data obtained from SliceOmatic.

All CT images contained between the endplates of the vertebrae directly above and below the fracture were in- 
cluded for analysis (Fig. 1A). For the fractured vertebra, all CT images containing the pedicles were analyzed.

$\mathrm{AD}$ of the vertebrae supra- and infra-adjacent to the fractured vertebra was measured at 10 regions of interest (ROI): eight independent ROI in the vertebral body and one for each pedicle. The eight ROI in the vertebral body consisted in these specific volumetric regions: (1) right superior anterior, (2) left superior anterior, (3) right inferior anterior, (4) left inferior anterior, (5) right superior posterior, (6) left superior posterior, (7) right inferior posterior, and (8) left inferior posterior. For the fractured vertebra, $\mathrm{AD}$ was measured at two ROI, which consist of both pedicles.

First, the entire vertebral body (cortical and cancellous bone) was identified and erosion (removal of one pixel width) was performed in each slice to remove the cortical shell of the vertebral body.

The vertebral body was then divided into four regions from the axial plane (Fig. 1B) by drawing two perpendicular lines. A first line bisects the vertebral body passing through and in line with the spinous process; two points on this line were identified, one at the most anterior part of the vertebral body and the other in the middle of the spinal canal. A second perpendicular line passes through the first line in the middle between the two points, thereby defining the right, left, anterior, and posterior margins for the ROI. Then, from the sagittal view, the superior and inferior margins of the ROI were defined as the vertebral body was divided in superior and inferior halves to obtain eight ROI.

Both pedicles were also first identified entirely (cancellous and cortical bone) and two erosions were performed at the pedicles to account for the wider cortical shell. This semi-automatic segmentation method generates eight

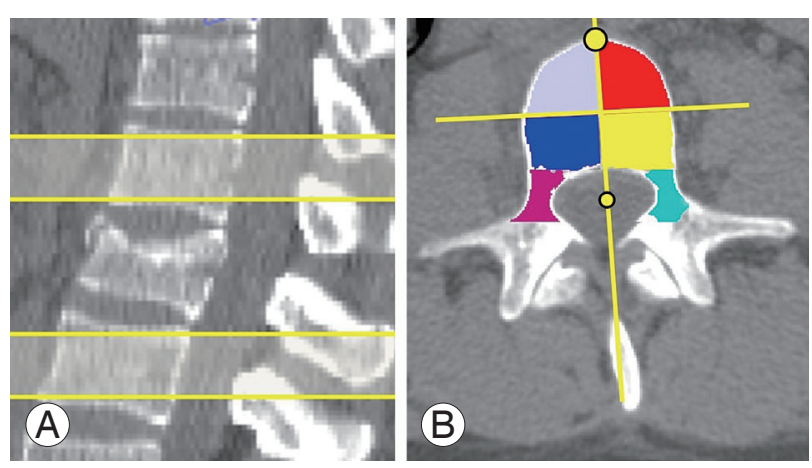

Fig. 1. (A) Identification of trabecular bone of superior and inferior vertebra in sagittal view. (B) Identification of region of interests in axial view.
ROI in the vertebral body and two ROI for the pedicles.

Based on the relationship between the average attenuation coefficient and the known density of calibration objects (Fig. 2), AD of all $10 \mathrm{ROI}$ at the vertebrae directly above and below the fractured vertebra was calculated. The AD for each ROI is calculated based on the attenuation coefficient of each voxel within the ROI. AD of the ROI for each intact pedicle at the level of the fractured vertebra is also calculated.

Overall, for each patient, $\mathrm{AD}$ was measured on $10 \mathrm{ROI}(8$ for the vertebral body and 2 for the pedicles) for vertebrae above and below the fracture, and 2 ROI (both pedicles) at the fracture level. For each patient, the $\mathrm{AD}$ of all $10 \mathrm{ROI}$ at the fractured vertebra was interpolated from the $\mathrm{AD}$ of vertebrae above and below the fractured vertebra, based on the assumption that $\mathrm{AD}$ at the fractured vertebra is linearly related to the $\mathrm{AD}$ of the vertebrae above and below the fracture.

\section{Data analysis}

The measured and interpolated $\mathrm{AD}$ for the pedicles of the fractured vertebra were compared using paired Student $t$ tests. Paired Student $t$ tests were also done to compare the $\mathrm{AD}$ between different $\mathrm{ROI}$ at the level of the fracture:

(1) Mean vertebral body $\mathrm{AD}$ (8 ROI) versus pedicles $\mathrm{AD}$ (2 ROI).

(2) Mean anterior $\mathrm{AD}$ (ROI) versus posterior $\mathrm{AD}$ (ROI) at the vertebral body.

(3) Mean superior versus inferior ROI.

(4) Mean right versus left ROI.

Pearson's correlation coefficient were used to study the

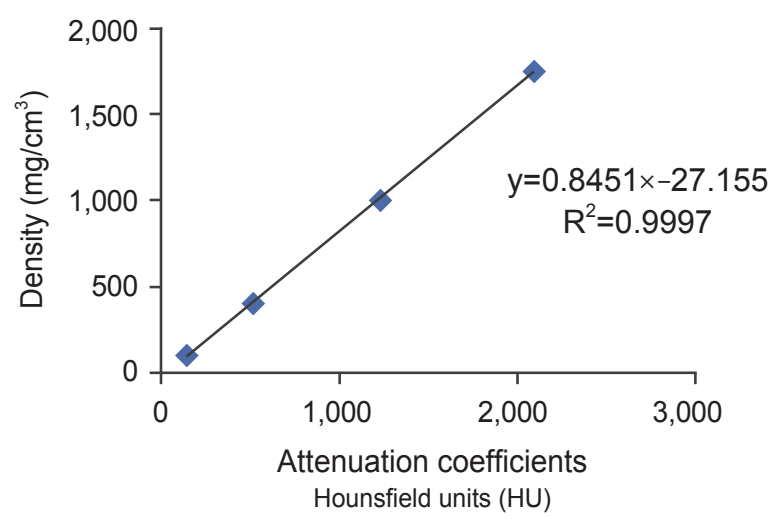

Fig. 2. The relation between the gray level and the known density of the calibration objects with the equations to be applied for region of interest (ROI); $R^{2}$, coefficient of determination. 
correlation between mean $\mathrm{AD}$ of each ROI and age. The significance level was set at 0.05. Data were examined with Statistica 6.1 software (StatSoft Inc., Tulsa, OK, USA).

\section{Results}

\section{Density of calibration objects}

The coefficient of determination $\left(\mathrm{R}^{2}\right)$ of relationships between the attenuation coefficients in CT images of the calibration objects of known density were 0.9997 , which indicates an excellent association (Fig. 2).

\section{Measured versus interpolated AD of pedicles at the fracture level}

The difference between the measured and the interpolated mean $\mathrm{AD}$ of the right and left pedicles at the fractured vertebra was 0.006 and $0.003 \mathrm{~g} / \mathrm{cm}^{3}$, which represents a difference of $2.0 \%$ and $1.0 \%$, respectively. The mean measured and estimated $\mathrm{AD}$ at the right pedicles were 0.296 $\mathrm{g} / \mathrm{cm}^{3}$ and $0.290 \mathrm{~g} / \mathrm{cm}^{3}$, the mean measured and estimated $\mathrm{AD}$ at the left pedicles were $0.289 \mathrm{~g} / \mathrm{cm}^{3}$ and $0.286 \mathrm{~g} / \mathrm{cm}^{3}$.

\section{Regional variation of $\mathrm{AD}$ within the fractured vertebra}

The mean estimated AD for each ROI of the fractured vertebra is presented in (Fig. 3).
The highest mean $\mathrm{AD}$ was found at the pedicles for the fractured vertebra; $0.296 \pm 0.07 \mathrm{~g} / \mathrm{cm}^{3}$ at the right pedicles and $0.289 \pm 0.067 \mathrm{~g} / \mathrm{cm}^{3}$ at left pedicles which is significantly $(p<0.01)$ higher than all ROI in the vertebral body $\left(0.155 \pm 0.041 \mathrm{~g} / \mathrm{cm}^{3}\right)(p<0.01)$.

The mean $\mathrm{AD}$ in posterior ROI $\left(0.166 \pm 0.044 \mathrm{~g} / \mathrm{cm}^{3}\right)$ was significantly higher $(p<0.01)$ than in the anterior ROI $\left(0.146 \pm 0.038 \mathrm{~g} / \mathrm{cm}^{3}\right)$ in the fractured vertebra.

AD was not significantly different $(p>0.01)$ between superior $\left(0.156 \pm 0.038 \mathrm{~g} / \mathrm{cm}^{3}\right)$ and inferior $(0.150 \pm 0.038 \mathrm{~g} /$ $\mathrm{cm}^{3}$ ) ROI of the fractured vertebral body.

There was no significant difference $(p>0.01)$ in $\mathrm{AD}$ between right $\left(0.182 \pm 0.06 \mathrm{~g} / \mathrm{cm}^{3}\right)$ and left $(0.182 \pm 0.06 \mathrm{~g} /$ $\mathrm{cm}^{3}$ ) ROI at the fractured vertebra, indicating the left and right sides of the vertebral bodies were symmetrical.

\section{Effect of age}

Pearson's correlation showed significant $(r=-0.72, p<0.01)$ negative correlation between age and estimated $\mathrm{AD}$ averaged for all eight ROI in the vertebral body at the fracture level. However, the correlation between age and estimated $\mathrm{AD}$ averaged for the two pedicles at the fractured vertebra was not significant $(r=-0.17, p>0.01)$.

\section{Discussion}

Once a vertebral fracture has occurred, it becomes impossible

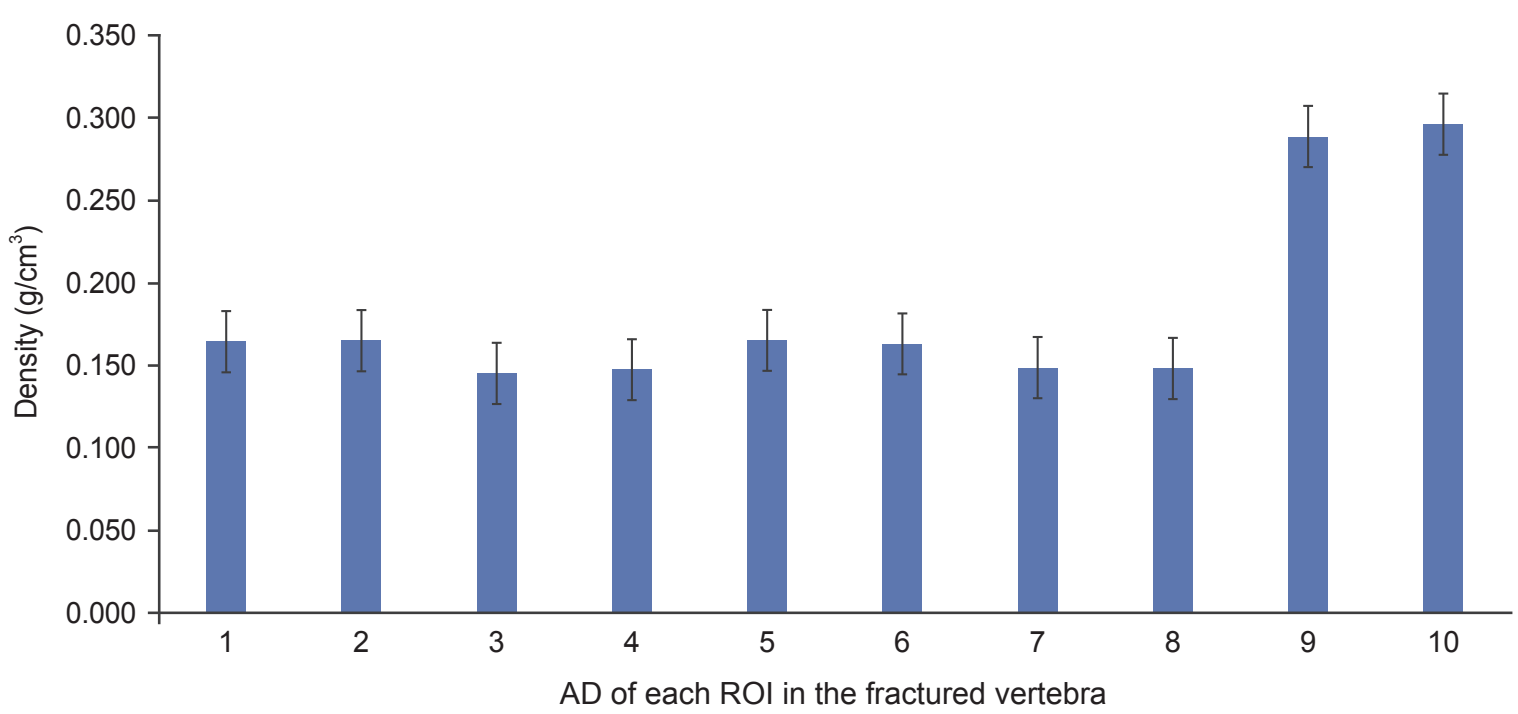

Fig. 3. Estimated apparent density (AD) (mean and standard deviation) of each region of interest (ROI) in the fractured vertebra. 1 , left inferior posterior, 2, right inferior posterior; 3, left inferior anterior; 4, right inferior anterior; 5, left superior posterior; 6 , right superior posterior; 7, left superior anterior; 8, right superior anterior; 9, left pedicle; 10, right pedicle. 
to measure the density of the fractured vertebra because of the alteration caused by the fracture itself. This study reports an innovative technique to assess the regional density in fractured vertebrae. The validity of interpolating the $\mathrm{AD}$ at the fracture level from the measured $\mathrm{AD}$ at the adjacent vertebrae is supported by our results showing that the difference between measured and interpolated mean $\mathrm{AD}$ of the pedicles at the fracture level was only 0.006 and $0.003 \mathrm{~g} / \mathrm{cm}^{3}$ for right and left pedicles respectively, which represents less than $2 \%$ of the measured $\mathrm{AD}$. In addition, the proposed technique uses QCT, discriminating the trabecular compartments, which is important since bone strength is substantially sensitive to a loss of trabecular bone $[15,18]$.

The results also showed that trabecular bone density is not homogeneous throughout the vertebra, as there is regional variation in density within the vertebrae [14]. This study shows that mean $\mathrm{AD}$ in the fractured vertebra was higher at the pedicles than at all ROI in the vertebral body, which is in agreement with previous studies $[19,20]$. Indeed, the stress concentration caused by an increase in $\mathrm{AD}$ at the pedicles when compared to the $\mathrm{AD}$ in the vertebral body is in accordance with the typical trapezoidal bony fragment retropulsing into the canal with burst fractures. Mean $\mathrm{AD}$ in posterior ROI was higher than in anterior ROI, which is also in agreement with previous studies [21]. These findings support the physiological importance of the middle column proposed in the classification of Denis $[22,23]$ and with the high occurrence of vertebral compression fractures associated with preservation of the middle column $[18,24]$. Whether a specific threshold in the variation in $\mathrm{AD}$ between the anterior (anterior ROI) and middle (posterior ROI) columns is associated with greater propensity of having a burst fracture rather than a compression fracture is not known but should be investigated in future studies.

Another important benefit of quantifying the $\mathrm{AD}$ of a fractured vertebra relates to the potential to estimate spinal stability and assess the risk of complications such as kyphosis, compression and/or neurological compromise following vertebral fracture. For clinicians, estimating spinal stability is essential to orient treatment, and assessing the $\mathrm{AD}$ of fractured vertebrae could help clinical choices.

As expected, the results showed significant a strong negative correlation between $\mathrm{AD}$ in the vertebral body and age, supporting a decline in bone strength with aging. On the opposite, the correlation between $\mathrm{AD}$ in the pedi- cles and age was not significant, in accordance with other studies showing that the decrease in BMD with aging varies from site to the other [13,25-27]. Whether the greater age-related AD decline in the vertebral body as compared to the pedicles predisposes the elderly population to compression fractures rather than burst fractures remains to be investigated in future biomechanical studies.

\section{Conclusions}

To our knowledge, this is the first study estimating the bone density of fractured vertebrae. It is proposed that $\mathrm{AD}$ at the fractured level can be accurately determined by interpolation from the adjacent vertebrae. This study also shows the presence of regional variations in vertebral bone density and the proportional decline in $\mathrm{AD}$ with age at the vertebral body but not in the pedicles. The proposed method could be used in a numerical model of the spine to better understand the mechanisms leading to various fracture patterns and evaluate the stability of the fracture.

\section{Conflict of Interest}

No potential conflict of interest relevant to this article was reported.

\section{References}

1. Briem D, Lehmann W, Ruecker AH, Windolf J, Rueger JM, Linhart W. Factors influencing the quality of life after burst fractures of the thoracolumbar transition. Arch Orthop Trauma Surg 2004;124:4618.

2. Briem D, Behechtnejad A, Ouchmaev A, et al. Pain regulation and health-related quality of life after thoracolumbar fractures of the spine. Eur Spine J 2007; 16:1925-33.

3. Lange U, Edeling S, Knop C, et al. Anterior vertebral body replacement with a titanium implant of adjustable height: a prospective clinical study. Eur Spine J 2007;16:161-72.

4. Vaccaro AR, Silber JS. Post-traumatic spinal deformity. Spine (Phila Pa 1976) 2001;26(24 Suppl):S111-8.

5. Wood KB, Bohn D, Mehbod A. Anterior versus posterior treatment of stable thoracolumbar burst fractures without neurologic deficit: a prospective, randomized study. J Spinal Disord Tech 2005;18 Suppl: 
S15-23.

6. Tabor Z, Rokita E. Quantifying anisotropy of trabecular bone from gray-level images. Bone 2007;40:96672.

7. Hui SL, Slemenda CW, Johnston CC Jr. Age and bone mass as predictors of fracture in a prospective study. J Clin Invest 1988;81:1804-9.

8. Small RE. Uses and limitations of bone mineral density measurements in the management of osteoporosis. MedGenMed 2005;7:3.

9. Ammann P, Rizzoli R. Bone strength and its determinants. Osteoporos Int 2003;14 Suppl 3:S13-8.

10. Cawte SA, Pearson D, Green DJ, Maslanka WB, Miller CG, Rogers AT. Cross-calibration, precision and patient dose measurements in preparation for clinical trials using dual energy X-ray absorptiometry of the lumbar spine. Br J Radiol 1999;72:354-62.

11. Bates DW, Black DM, Cummings SR. Clinical use of bone densitometry: clinical applications. JAMA 2002; 288:1898-900.

12. Engelke K, Adams JE, Armbrecht G, et al. Clinical use of quantitative computed tomography and peripheral quantitative computed tomography in the management of osteoporosis in adults: the 2007 ISCD Official Positions. J Clin Densitom 2008;11:123-62.

13. Hulme PA, Boyd SK, Ferguson SJ. Regional variation in vertebral bone morphology and its contribution to vertebral fracture strength. Bone 2007;41:946-57.

14. Guo XE, Kim CH. Mechanical consequence of trabecular bone loss and its treatment: a three-dimensional model simulation. Bone 2002;30:404-11.

15. Parfitt AM, Mathews CH, Villanueva AR, Kleerekoper M, Frame B, Rao DS. Relationships between surface, volume, and thickness of iliac trabecular bone in aging and in osteoporosis. Implications for the microanatomic and cellular mechanisms of bone loss. J Clin Invest 1983;72:1396-409.

16. Ito M, Hayashi K, Yamada M, Nakamura T. Vertebral measurements for assessment of osteoporosis. Br J Radiol 1994;67:759-63.
17. Hordon LD, Raisi M, Aaron JE, Paxton SK, Beneton M, Kanis JA. Trabecular architecture in women and men of similar bone mass with and without vertebral fracture: I. Two-dimensional histology. Bone 2000;27: 271-6.

18. Inceoglu S, Burghardt A, Akbay A, Majumdar S, McLain RF. Trabecular architecture of lumbar vertebral pedicle. Spine (Phila Pa 1976) 2005;30:1485-90.

19. Tanck E, Homminga J, van Lenthe GH, Huiskes R. Increase in bone volume fraction precedes architectural adaptation in growing bone. Bone 2001;28:6504.

20. van Lenthe GH, Huiskes R. How morphology predicts mechanical properties of trabecular structures depends on intra-specimen trabecular thickness variations. J Biomech 2002;35:1191-7.

21. Dai LY, Wang XY, Wang CG, Jiang LS, Xu HZ. Bone mineral density of the thoracolumbar spine in relation to burst fractures: a quantitative computed tomography study. Eur Spine J 2006;15:1817-22.

22. Denis F. The three column spine and its significance in the classification of acute thoracolumbar spinal injuries. Spine (Phila Pa 1976) 1983;8:817-31.

23. Denis F. Spinal instability as defined by the threecolumn spine concept in acute spinal trauma. Clin Orthop Relat Res 1984;(189):65-76.

24. Melton LJ 3rd, Lane AW, Cooper C, Eastell R, O'Fallon WM, Riggs BL. Prevalence and incidence of vertebral deformities. Osteoporos Int 1993;3:113-9.

25. Bell GH, Dunbar O, Beck JS, Gibb A. Variations in strength of vertebrae with age and their relation to osteoporosis. Calcif Tissue Res 1967;1:75-86.

26. Weaver JK, Chalmers J. Cancellous bone: its strength and changes with aging and an evaluation of some methods for measuring its mineral content. J Bone Joint Surg Am 1966;48:289-98.

27. Silva MJ, Gibson LJ. Modeling the mechanical behavior of vertebral trabecular bone: effects of age-related changes in microstructure. Bone 1997;21:191-9. 